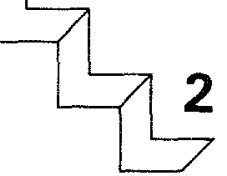

\title{
An economic approach to social choice - II
}

\section{Theodore Bergstrom and Trout Rader*}

\begin{abstract}
There is a formal equivalence between games, societies, and economies. Lindahl equilibrium for a game or society corresponds to competitive equilibrium for the equivalent economy. Results on existence and optimality of competitive equilibrium thus apply to the theory of games and societies. The "core" for a game or society as derived by extension from the core of an economy is "too large" to be interesting. An example illustrates that the $\alpha$-core may be disjoint from the set of Lindahl equilibria. However, if the power of coalitions to inflict negative externalities is suitably restricted, Lindahl equilibria must be in the $\alpha$-core.
\end{abstract}

\section{Introduction}

The fact that an economy with public goods may be treated as a private goods economy in a commodity space of higher dimension was noted by Arrow (1969) and later exploited by others, including Starrett (1973), Rader (1972) and Bergstrom (1976). Here we develop this notion systematically by demonstrating the equivalence of the notions of game, society, and economy. This enables us to define Lindahl equilibrium for a game or society in such a way as to correspond to competitive equilibrium for the equivalent economy. In view of this equivalence, theorems on the existence of Lindahl equilibrium become straightforward corollaries of theorems on the existence of competitive equilibria for the corresponding economies. We show that the notion of a core for a game or society derived by extension from the core of the equivalent economy is relatively uninteresting since it is "too large." We then study the $\alpha$-core of Scarf (1971) for a game or society. We show by example that the $\alpha$-core and the set of Lindahl equilibria may be disjoint. This extends and sharpens the observation of Shapley and Shubik (1969) and Bergstrom (1975) that some

*University of Michigan and Washington University, St. Louis, respectively. 


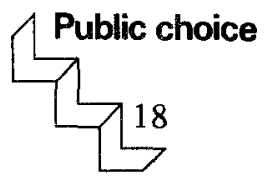

Lindahl equilibria are not in the $\alpha$-core. We also extend results of Shapley and Shubik (1969), Starrett (1973) and Bergstrom (1976), by demonstrating that the Lindahl equilibrium is in the core when the power of coalitions to impose negative externalities from equilibrium is removed.

\section{Models of social interaction}

We discuss three alternative models of social interaction. These are a game, a society, and an economy. The notions of game and economy are familiar. Our discussion motivates the notion of a society. It will be shown that all three concepts are related by isomorphism.

Definition 1 . Where $L^{g}$ is a linear space, an $n$ person game $\left(Y_{1}, \ldots, Y_{n}\right.$, $R_{1}^{g}, \ldots, R_{n}^{g}$ ) on $L^{g}$ is characterized by the following sets:

1. For $i=1, \ldots, n$, the strategy set of Agent $i$ is $Y_{i} \subset L^{g}$ where $0 \in Y_{i}{ }^{1}$

2. The outcome set is $Y=\underset{i=1}{n} Y_{i}{ }^{2}$

3. For $i=1, \ldots, n$, the preference relation of Agent $i$ on outcomes is $R_{i}^{g} \subset Y \times Y$.

Definition 2. Where $L^{s}$ is a linear space, an $n$ person society $\left(X_{1}, \ldots, X_{n}\right.$, $R_{1}^{s}, \ldots, R_{n}^{s}$ ) on $L^{s}$ is characterized by the following sets:

1. For $i=1, \ldots, n$, the action set of Agent $i$ is $X_{i} \subset L^{s}$ where $0 \in X_{i}{ }^{3}$

2. The set of social states is $X=\sum_{i=1}^{n} X_{i}{ }^{4}$

3. For $i=1, \ldots, n$, the preference relation of Agent $i$ on social states is $R_{i}^{s} \subset X \times X$

(Literally, a society is the sum of its parts.)

An example of a society is the following. Imagine a village with $n$ citizens each of whom pursues the sole activity of driving an automobile through the village streets. Although each citizen loves to drive, they all abhor the congestion and pollution due to traffic. Assume that each citizen does not care which of his fellow citizens are driving, so long as the total amount of driving by others is fixed. For each $i$, suppose that any possible amount of driving by $i$ can be represented by a number in the closed real interval $\left[0, a_{i}\right]$.

To model our village as a society we represent the action of a citizen $i$ who drives $z_{1}$ hours by an $n+1$ vector $x_{1}$ with an entry of $z_{1}$ in the ith 
coordinate and in the $n+1$ coordinate with zeroes elsewhere. Then define the action set of $i$ as $X_{i}=\left\{\left(0, \ldots, 0, z_{i}, 0, \ldots, 0, z_{i}\right) \in R^{n+2} 0 \leqq z_{i} \leqq a_{i}\right\}$. Then for any social state $x \in \Sigma X_{i}$, the ith component reports the amount of driving by $i$ while the $n+1$ component reports the total amount of driving summed over all citizens. Thus preference levels are fully specified by the choice of a point in $X=\Sigma X_{i}$. In fact, the preference relation $R_{i}^{s}$ will in this example have the property that $R_{i}^{s} \supset\left\{(y, z) \in X \times X \mid y_{i} \geqq z_{i}\right.$, $\left.y_{n+1} \leq z_{n+1}\right\}$.

Alternatively village affairs could be modelled as a game in which for each $i, Y_{i}=\left[0, a_{i}\right], Y=\underset{i=1}{\stackrel{n}{\times}} Y_{i}$ and where $\left(y_{1}, \ldots, y_{n}\right) R_{i}^{g}\left(y_{1}^{\prime}, \ldots, y_{n}^{\prime}\right)$ if and only if $\left(y_{i}, x\right) R_{i}^{s}\left(y_{i}^{\prime}, x^{\prime}\right)$ where $x=\sum_{j=1}^{n} y_{i}$, and $x^{\prime}=\sum_{j=1}^{n} y_{j}^{\prime}$. It is easily seen that this procedure can be generalized as follows.

Remark 1. There is a one to one mapping from the set of $n$ person societies on a linear space $L^{s}$ into (but not onto) the set of $n$ person games on $L^{g}=L^{s}$ such that the society $\left(X_{1}, \ldots, X_{n}, R_{1}^{s}, \ldots, R_{n}^{s}\right)$ is identified with the game $\left(Y_{1}, \ldots, Y_{n}, R_{1}^{g}, \ldots R_{n}^{g}\right)$ where:

1. $Y_{i}=X_{i}$

2. $Y=\stackrel{n}{\underset{x}{=}} Y_{i}=\stackrel{n}{x}_{i=1}^{n} X_{i}$.

3. $R_{i}^{g}=\left\{\left(y_{1}, \ldots, y_{n}\right),\left(y_{1}^{\prime}, \ldots, y_{n}^{\prime}\right) \epsilon Y \times Y \mid \Sigma y_{i} R_{i}^{s} \Sigma y_{1}^{\prime}\right\}$.

Slightly less obvious is the fact that with an appropriate expansion of the dimension of the space in which it is embedded, a game can be regarded as a society. This is formalized below.

Remark 2. There is a one to one mapping from the set of $n$ person games on a linear space $L^{g}$ into the set of $n$ person societies on $\left(L^{g}\right)^{n}$ (the $n$-fold Cartesian product of $\left.L^{g}\right)$, such that the game $\left(Y_{1}, \ldots, Y_{n}, R_{1}^{g}, \ldots, R_{n}^{g}\right)$ is identified with the society $\left(X_{1}, \ldots, X_{n}, R_{1}^{s}, \ldots, R_{n}^{s}\right)$ where:

1. $X_{i}=\left\{\left(0, \ldots, x_{i}, \ldots, 0\right) \in\left(L^{g}\right)^{n} \mid x_{i} \in Y_{i}\right\}$.

2. $X=\sum_{i=1}^{n} X_{i}=\stackrel{n}{x}_{i=1}^{n} Y_{i}=Y$

3. $R_{i}^{s}=R_{i}^{g}$

Rather surprisingly, it happens that the concept of an economy is at least as 


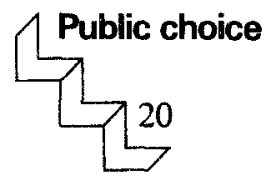

general as that of a game or a society. Our definition of an economy is similar to that of Debreu (1959). However we attach production possibility sets directly to individuals rather than to firms whose ownership is partitioned among individuals. Where production possibility sets are convex, these two approaches are equivalent, as has been observed by Rader (1972) and Nikaido (1968). The procedure used here leads to a less cumbersome treatment of game theoretic notions.

Definition 3. Where $L^{e}$ is a linear space, an $n$ person economy $\left(C_{1}, \ldots, C_{n}\right.$, $T_{1}, \ldots, T_{n}, R_{1}, \ldots, R_{n}$ ) on $L^{e}$ is characterized by the following sets:

1. For $i=1, \ldots, n$, the consumption set of Agent $i$ is $C_{i} \subset L^{e}$ and the production possibility set of Agent $i$ is $T_{i} \subset L^{e}$.

2. The set of feasible allocations is $Z=\underset{i=1}{x_{=}^{n}} C_{i} \cap\left\{\left(z_{1}, \ldots, z_{n}\right) \mid \sum_{i=1}^{n}\right.$ $\left.z_{i} \in \Sigma T_{i}\right\}$

3. For $i=1, \ldots, n$, the preference relation of Agent $i$ is $R_{i}^{e} \subset C_{i} \times C_{i}$.

Condition (3) deserves further explanation. The total consumption of the economy is $\Sigma z_{i}$. According to conditon (3), the total consumption must be equal to the sum of some production in the economy. In effect, exchange of commodities entails no cost.

It turns out that every society and every game is "equivalent to" an economy. This is the basic result of Rader (1973) and is stated formally as remarks 3 and 4 . We also examine the somewhat tangential but very interesting question of when an economy is equivalent to a society or game. Remarks 5 and 6 contains an answer to this question. Verification of each of these remarks is straightforward.

Remark 3. There exists a one to one mapping from the set of $n$ person societies on $L^{s}$ into the set of $n$ person economies on $\left(L^{s}\right)^{n}$ such that the society $\left(X_{1}, \ldots, X_{n}, R_{1}^{s}, \ldots, R_{n}^{s}\right)$ is identified with the economy $\left(C_{1}, \ldots, C_{n}, T_{1}, \ldots, T_{n}, R_{1}^{e}, \ldots, R_{n}^{e}\right)$ where:

1. $C_{i}=\left\{\left(x_{1}, \ldots, x_{n}\right) \epsilon\left(\Sigma X_{i}\right)^{n} \mid x_{j}=0\right.$ for $\left.j \neq i\right\}$

$T_{i}=\left\{\left(x_{1}, \ldots, x_{n}\right) \epsilon\left(X_{i}\right)^{n} \mid x_{1}=x_{2}=\ldots=x_{n}\right\}$.

2. $Z=\underset{i=1}{\stackrel{n}{x}} C_{i} \cap\left\{\left(z_{1}, \ldots, z_{n}\right) \mid \Sigma z_{j} \in \Sigma T_{j}\right\}$.

$=\left\{\left(z_{1}, \ldots, z_{n}\right) \mid\right.$ for some $x \in \Sigma X_{j}$ and all $i=1, \ldots, n, z_{i}$

$\left.=(0, \ldots, x, \ldots, 0) \in C_{i}\right\}$. 
3. $R_{i}^{e}=\left\{\left(x_{1}, \ldots, x_{n}\right),\left(x_{1}^{\prime}, \ldots, x_{n}^{\prime}\right) \in C_{i} x C_{i} \mid\left(x_{i} x_{i}^{\prime}\right) \in R_{i}^{s}\right\}$.

Remark 4. There exists a one to one mapping from the set of $n$ person games on $L^{g}$ into the set of $n$ person economies on $\left(L^{g}\right)^{n^{2}}$ such that the game $\left(Y_{1}, \ldots, Y_{n}, R_{1}, \ldots, R_{n}\right)$ is identified with the economy $\left(C_{1}, \ldots, C_{n}, T_{1}, \ldots, T_{n}, R_{1}, \ldots, R_{n}\right)$ where:

1. $C_{i}=\left\{\left(z_{1}, \ldots, z_{n}\right) \in(Y)^{n} \mid z_{j}=0\right.$ for $j \neq i$ and $\left.z_{i} \in Y\right\}$.

$T_{i}=\left\{\left(z_{1}, \ldots, z_{n}\right) \epsilon(Y)^{n} \mid\right.$ for some $y_{i} \in Y_{i}, z_{j}=\left(0, \ldots, y_{i}, \ldots, 0\right) \epsilon Y$ for all $j=1, \ldots, n\}$.

2. $Z=\underset{i=1}{\stackrel{n}{\times}} C_{i} \cap\left\{\left(z_{1}, \ldots, z_{n}\right) \epsilon(Y)^{n} \mid \sum_{i=1}^{n} z_{i} \epsilon \sum_{i=1}^{n} T_{i}\right\}=\left\{\left(z_{1}, \ldots, z_{n}\right)\right.$ $\epsilon(Y)^{n} \mid$ for some $y \in Y, z_{i}=y$ for all $\left.i=1, \ldots, n\right\}$.

3. $R_{i}^{e}=\left\{\left(\left(z_{1}, \ldots, z_{n}\right),\left(z_{1}^{\prime}, \ldots, z_{n}^{\prime}\right)\right) \in C_{i} \times C_{i} \mid\left(z_{i}, z_{i}^{\prime}\right) \in R_{i}^{g}\right\}$.

The obvious way to "make an economy into a society" is to allow individuals to "allocate" their production among the agents. Thus for each $i$ we might make $X_{i}=\left\{\left(z_{i 1}, \ldots, z_{j n}\right) \mid \frac{\Sigma}{j} z_{i j} \in T_{i}\right\}$ where $z_{i j}$ is the amount of $i$ 's production allocated to $j$. For $x \in \Sigma X_{i}$, the $j$ th component $X_{j}$ of $X$ would represent the total vector of production allocated to $j$. Thus it would be natural to assign preferences $R_{i}^{s}$ so that $x R_{i}^{s} x^{\prime}$ if and only if $x_{i} R_{i}^{e} x_{i}^{\prime}$. The only snag here is that for this procedure to work smoothly we must ensure that $x_{i}$ and $x_{i}^{\prime}$ belong to the consumption set $C_{i}^{e}$. This we achieve by allowing only non-negative transfers and by assuming that each $C_{i}^{e}$ is the nonnegative orthant of $L^{e}$ so that no individual can be "forced out of his consumption set" by admissable transfers from others. Remarks 5 and 6 explicate the situation formally.

Remark S. Let $L_{+}^{e}$ be the non-negative orthant of an ordered linear space. There is a one to one mapping from the set of $n$ person economies on $L^{e}$ for which $C_{i}=L_{+}^{e}$ for $i=1, \ldots, n$ into the set of societies on $\left(L^{e}\right)^{n}$ such that the economy $\left(C_{1}, \ldots, C_{n}, T_{1}, \ldots, T_{n}, R_{1}^{e}, \ldots, R_{n}^{e}\right)$ is identified with the society $\left(X_{1}, \ldots, X_{n}, R_{1}^{s}, \ldots, R_{n}^{s}\right)$ where:

1. $X_{i}=\left\{z_{i} \epsilon\left(L_{+}^{e}\right)^{n} \mid z_{i}=\left(z_{i 1}, \ldots, z_{i n}\right)\right.$ and $\left.\sum_{j=1}^{n} z_{i j} \epsilon T_{i}\right\}$.

2. $X=\sum_{i=1}^{n} X_{i}$

3. $R_{i}^{s}=\left\{\left(x, x^{\prime}\right) \in X \times X \mid\left(x_{i}, x_{i}^{\prime}\right) \in R_{i .}^{e}\right\}$. 


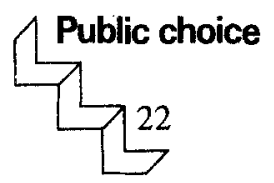

Remark 6. Let $L_{+}^{e}$ be the non-negative orthant of an ordered linear space. There is a one to one mapping from the set of $n$ person economies on $L^{e}$ for which $C_{i}=L_{+}^{e}$ for $i=1, \ldots, n$ into the set of games on $\left(L^{e}\right)^{n}$ such that the economy $\left(C_{1}, \ldots, C_{n}, T_{1}, \ldots, T_{n}, R_{1}^{e}, \ldots, R_{n}^{e}\right)$ is identified with the game $\left(Y_{1}, \ldots, Y_{n}, R_{1}^{g}, \ldots, R_{n}^{g}\right)$ where:

1. $Y_{i}=\left\{z_{i} \epsilon\left(L_{+}^{e}\right)^{n} \mid z_{i}=\left(z_{i 1}, \ldots, z_{i n}\right)\right.$ and $\left.\sum_{j=1}^{n} z_{i j} \in T_{i}\right\}$,

2. $Y=\stackrel{n}{\underset{x}{x}} Y_{i}$

3. $R_{i}^{g}=\left\{\left(z_{1}, \ldots, z_{n}\right),\left(z_{1}^{\prime}, \ldots, z_{n}^{\prime}\right) \in\left(L^{e}\right)^{n} \times\left(L^{e}\right)^{n} \mid\left(\sum_{i=1}^{n} z_{i}\right.\right.$,

$$
\left.\left.\sum_{i=1}^{n} z_{i}^{\prime}\right) \in R_{i}^{s}\right\}
$$

\section{Competitive equilibrium and Lindahl equilibrium.}

The equivalence of economies, games, and societies enables us to extend results in competitive theory to the theory of games and societies.

Definition 4. A competitive equilibrium for the economy $\left(C_{1}, \ldots, C_{n}\right.$, $\left.T_{1}, \ldots, T_{n}, R_{1}^{e}, \ldots, R_{n}^{e}\right)$ on $L^{e}$ consists of points $\left(\bar{x}_{1}, \ldots, \bar{x}_{n}\right) \varepsilon$ $i \stackrel{n}{\stackrel{x}{=}} C_{i}$ and $\bar{p} \epsilon\left(L^{e}\right)^{*}$ (where $\left(L^{e}\right)^{*}$ is the dual space of $\left.\left(L^{e}\right)\right)$ such that:

1. For each $i, \bar{x}_{i}$ maximizes $R_{i}^{e}$ on the budget set $\left\{x_{i} \in C_{i} \mid, \bar{p} x_{i} \leqq \max _{z \in T_{i}} \bar{p} z\right\}$. 2. $\sum_{i=1}^{n} \bar{x}_{i} \in \sum_{i=1}^{n} T_{i}$

Definition 5. A Lindahl equilibrium for a society $\left(X_{1}, \ldots, X_{n}, R_{1}^{s}, \ldots, R_{n}^{s}\right)$ on $L^{s}$ consists of points $\bar{x}_{i} \in X_{i}$ and $\bar{p}^{i} \in\left(L^{s}\right)^{*}$ (the dual space of $L^{s}$ ) for each $i$ such that $\bar{x}={ }_{i}^{\Sigma} \bar{x}_{i}$ optimizes $R_{i}^{s}$ on $\left\{x \in X \mid \bar{p}^{i} x \leqq \max _{x_{i} \in X_{i}} \bar{p} x_{i}\right\}$.

Definition 6. A Lindahi equilibrium for a game $\left(Y_{1}, \ldots, Y_{n}, R_{1}, \ldots, R_{n}\right)$ on $L^{g}$ consists of points $\bar{y}_{i} \in Y_{i g}\left(\bar{p}_{1}^{i}, \ldots \bar{p}_{n}^{i}\right) \in\left(\left(L^{g}\right)^{n}\right)^{*}$ (the dual space of the $n$-fold Cartesian product of $\left.L^{g}\right)$ such that for each $i, \bar{y}=\left(\bar{y}_{1}, \ldots, \bar{y}_{n}\right)$ $\operatorname{maximizes} R_{i}^{g}$ on $\left\{\left(y_{1}, \ldots, y_{n}\right) \in Y \mid \sum_{j=1}^{n} \bar{p}_{j}^{i} y_{j} \leqq \sum_{j=1}^{n} \bar{p}_{i}^{j} y_{i}\right\}$. 
In Lindahl equilibrium for either a society or a game, each individual has his own price vector used to evaluate the final social state. His "budget set" consists of those social states whose "cost" at his own prices does not exceed the value of his own activity valued at the sum of the prices of all agents.

Remark 7. If a society or a game is equivalent to an economy as in remark 3 or remark 4 , then a competitive equilibrium for the economy is a Lindahl equilibrium for the game or society.

Remark 7 can be verified as a direct consequence of the definitions. An immediate consequence of remark 7 is:

Remark 8. If a society or game is equivalent to an economy for which competitive equilibrium exists, then there exists a Lindahl equilibrium for the society or game.

We can extend the fundamental results on the relation between Pareto optima and competitive equilibria for economies to that between Pareto optima and Lindahl equilibria for games and sócieties. By direct application of the technique used to prove the optimality of competitive equilibrium we have:

Remark 9. If preferences of all agents are locally non-satiated, a Lindahl equilibrium for a game or a society is Pareto optimal.

It is easy to show that a Pareto optimal outcome or social state for a game or a society corresponds to a Pareto optimum for the equivalent economy. If the "second theorem of welfare economics" applies to the equivalent economy, then this Pareto optimum for the economy is a competitive equilibrium given an appropriate distribution of wealth. It is easy to show as a consequence of Remark 7 that the original Pareto optimum for the game or social state is also a Lindahl equilibrium for a game or social state obtained by a transformation of the origin of the original game or social state. This result is made explicit as:

Remark 10. If a game or society is equivalent to an economy for which the "second theorem of welfare economics" applies, then any Pareto optimal outcome or social state for the game or society is a Lindahl equilibrium outcome or social state for the game or society obtained by translating the coordinates of the original game or society so that the specified Pareto optimum becomes the origin.

Since the equivalence mappings of Remarks 3 and 4 preserve convexity and closedness of sets in a linear topological space, the equivalent economy will inherit properties of convexity and continuity assumed for a game or society. Therefore in a game or a society, we assume continuity and convexity of preferences and closedness and convexity of strategy sets or actions sets, 


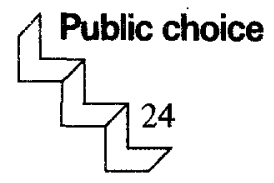

along with certain technical assumptions to deal with "boundary problems", then we can be assured that in the equivalent economy, competitive equilibrium will exist and the second theorem of welfare economics will hold. Thus the applicability of remarks 9 and 10 is established. These matters are treated in Rader (1973) and in more detail in Bergstrom (1976).

IV. The "core" in economies, games, and societies

A. The "core" as extended from an equivalent economy

A well known result of Shapley (in Rader, 1972, or Nikaido, 1968) is that every competitive equilibrium belongs to the core of an economy. If the core of a game or society were defined to consist of those outcomes or social situations that correspond under the mappings of Remarks 3 and 4 to points in the core of the equivalent economy, then the Lindahl equilibria which according to Remarks 7 and 8 correspond to the competitive equilibria would belong to the core. This procedure, however, leads to a core which is too large to be of interest. Indeed it turns out that the core would then contain all Pareto optima which are Pareto superior to the outcome in which all agents undertake the zero strategy. This result is stated formally as Remark 11 for the case of a society. A similar remark applies to games.

Definition 7. Let $\left(C_{1}, \ldots, C_{n}, T_{1}, \ldots, T_{n}, R_{1}, \ldots, R_{n}\right)$ be an economy. Where $K \subset\{1, \ldots, n\}$, coalition $K$ is said to be able to improve on an allocation $\left(x_{1}, \ldots, x_{n}\right) \in \underset{i=1}{\stackrel{n}{\times}} C_{i}$ if there exists $\left(x_{1}^{\prime}, \ldots, x_{n}^{\prime}\right) \in \underset{i=1}{\stackrel{n}{\times}} C_{i}$ such that $\underset{j \in K}{\Sigma} x_{j}^{\prime} \in \underset{j \in K}{\Sigma} T_{j}$ and $x_{j}^{\prime} R_{j} x_{j}$ for all $j \in K$ while $x_{i}^{\prime} P_{i} x_{i}$ for some $i \in K$. The core of the economy is the set of allocations which are feasible and which can be improved on by no coalition $K \subset\{1, \ldots, n\}$.

Remark 11. If a Society $S$ is equivalent to an economy $E$, then the set of social states in $S$ which correspond to allocations in the core of $E$ is the set of Pareto optimal social states $x \in S$ such that $x R_{i}^{s} 0$ for all $i \in I$.

The proof of Remark 11 is straightforward once it is observed from the construction of the sets $C_{i}$ and $T_{i}$ in the equivalent economy that for any coalition $K \subsetneq\{1, \ldots, n\}, \underset{j \in K}{\Sigma} C_{j} \cap \underset{j \in K}{\Sigma} T_{j}=0$. Thus coalitions other than $\{1, \ldots, n\}$ are unable to improve on the situation in which each agent undertakes the zero activity.

\section{B. The $\alpha$-core}

In view of Remark 11, we must look elsewhere for an interesting notion of the "core" of a game or society. One such alternative, the $\alpha$-core, is due to Scarf (1971). In order to improve on allocation in the sense of the $\alpha$ core, 
a coalition must be able to assign alternative strategies to its members in such a way that regardless of the responses in the complementary coalition, no member of the coalition is made worse off and at least one member benefits. This is formalized as follows.

Definition 8. Let $\left(Y_{1}, \ldots, Y_{n}, R_{1}^{g}, \ldots, R_{n}^{g}\right)$ be an $n$ person game. Coalition $K \subset\{1, \ldots, n\}$ is able to improve (in the sense of the $\alpha$-core) on the outcome $\left(y_{1}, \ldots, y_{n}\right) \in Y$ if there exists $\hat{y}_{j} \in Y_{j}$ for each $j \in K$ such that for all $y^{\prime} \in Y \cap\left\{\left(y_{1}^{\prime}, \ldots, y_{n}^{\prime}\right) \mid y_{j}^{\prime}=\hat{y}_{j}\right.$ for all $\left.j \in K\right\}, y^{\prime} R_{j} y$ for all $j \in K$ and $y^{\prime} P_{i} y$ for some $i \in K$. The $\alpha$-core is the set of outcomes in $Y$ on which no coalition $K \subset\{1, \ldots, n\}$ can improve.

Definition 9. Let $\left(X_{1}, \ldots, X_{n}, R_{1}^{s}, \ldots, R_{n}^{s}\right)$ be an $n$ person society. Coalition $K \subset\{1, \ldots, n\}$ is able to improve on $\left(x_{1}, \ldots, x_{n}\right) \in \underset{i=1}{\stackrel{n}{x} X_{i}}$ if there exists $\hat{x}_{j} \in X_{j}$ for each $j \in K$ such that for all $x^{\prime} \in \underset{j \notin K}{\sum_{j}} X_{j}, \sum_{j \in K} \hat{x}_{j}+x^{\prime}$ $R_{i}^{s} \sum_{j=1}^{n} x_{j}$ for all $i \in K$ with strict preference for some $i \in K$. The $\alpha$-core is the set of social states on which no coalition can improve.

Scarf shows that where $Y$ is convex and compact and where each $R_{i}^{g}$ represents continuous, convex preferences the $\alpha$ core is non empty. In view of remark 1 and Scarf's result we can assert:

Remark 12. In a society where for all $i \in I, X_{i}$ is convex and compact and $R_{i}^{s}$ represents continuous, convex preferences, the $\alpha$ core is non-empty.

The $\alpha$ core, however, has at least two features which are undesirable for our purposes. Let the game be one in which many agents are able to inflict severe damages on others. Then the conservative nature of the blocking criterion is such that the $\alpha$ core turns out to be little less than the set of Pareto optimal social states. In fact the $\alpha$ core may even include social states which are worse for some agents than the initial state $0 \epsilon X$. Also, where there are asymmetries in the possibilities for agents to damage others, the $\alpha$ core may exclude the Lindahl equilibrium social states. The difficulties are illustrated in the following example.

\section{Example 1}

This example can be thought of as a two person game in which each participant performs an activity which he enjoys but which distresses the other. Formally, let $Y_{1}$ and $Y_{2}$ be the closed real intervals $[0, a]$ and $[0, b]$. Preferences of Agents 1 and 2 are represented by the utility functions, $u^{1}\left(y_{1}, y_{2}\right)=y_{1}-\frac{1}{2} y_{2}^{2}$ and $u^{2}\left(y_{1}, y_{2}\right)=y_{2}-\frac{1}{2} y_{1}^{2}$. It is not hard to show 


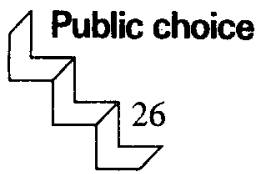

that the set of Pareto optimal outcomes is: $\left\{\left(y_{1}, y_{2}\right) \mid y_{1} \cdot y_{2}=1\right.$, $\left.0 \leqq y_{1} \leqq a, 0 \leqq y_{2} \leqq b\right\} \cup\left\{\left(a, y_{2}\right) \mid 0 \leqq y_{2} \leqq \frac{1}{a}\right\} \cup\left\{\left(y_{1}, b\right) \mid 0 \leqq y_{1} \leqq \frac{1}{h}\right\}$.

To find the $\alpha$-core, we observe that the highest utility which Agent 1 can guarantee himself is $u^{1}(a, b)=a-\frac{1}{2} b^{2}$ while the best that Agent 2 can guarantee himself is $b-\frac{1}{2} a^{2}$. Thus the $\alpha$-core consists of those Pareto optimal outcomes $\left(y_{1}, y_{2}\right)$ such that $u^{1}\left(y_{1}, y_{2}\right) \geqq a-\frac{1}{2} b^{2}$ and $u^{2}\left(y_{1}, y_{2}\right)$ $\geqq b-\frac{1}{2} a^{2}$. These limits will both be negative for appiopriate choices of $a$ and $b$. (For example, let $a=b>2$ ). Thus the $\alpha$-core can contain outcomes which each agent regards as interior to the "initial state" $(0,0)$. It is also clear that the $\alpha$-core can be made arbitrarily large by choice of sufficiently large values of $\underline{a}$ and $\underline{b}$.

Where $a \geqq 1$ and $b \geqq 1$, there is a Lindahl equilibrium in which the Lindah1 prices are $p^{1}=(1,-1)$ and $p^{2}=(-1,1)$ and the strategies are $y_{1}=y_{2}=1$. To see that this is a Lindahl equilibrium, notice that $(1,1)$ maximizes $u^{2}\left(y_{1}\right.$,

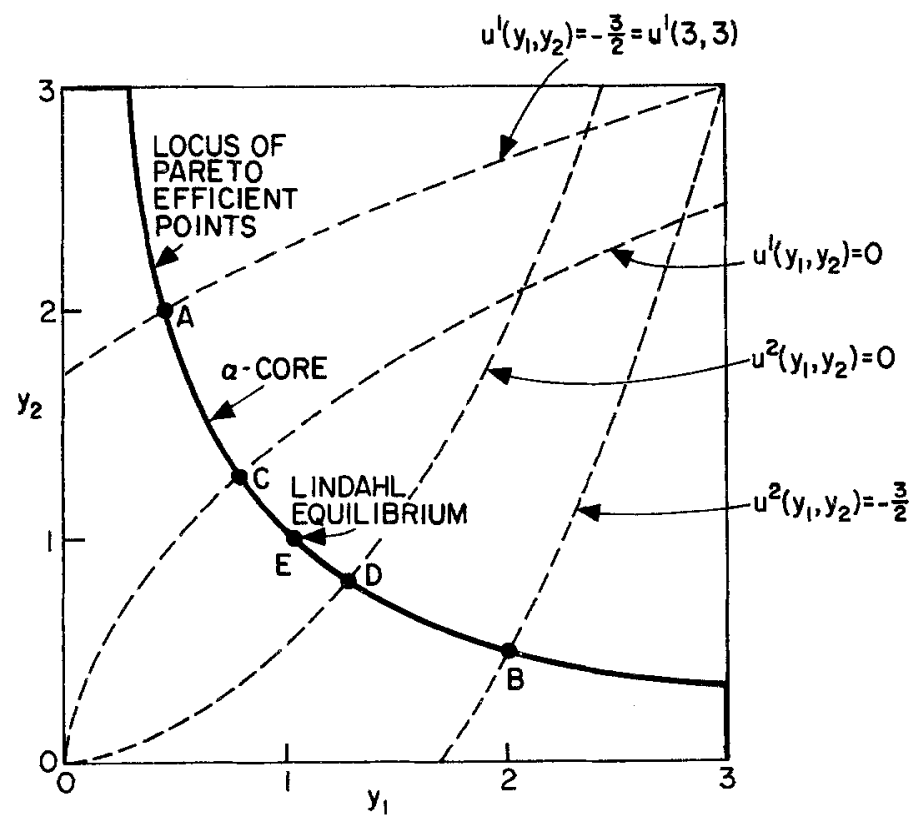

Figure 1. 
$\left.y_{2}\right)$ subject to the constraint $1 \cdot y_{1}+(-1) y_{2} \leqq \max _{y_{1} \in[0, a]}\left[p_{1}^{1}+p_{1}^{2}\right] y_{1}=$ $\max _{y_{2} \in[0, a]}[1+(-1)] y_{1}=0$ and that likewise $(1,1)$ maximizes $u^{2}\left(y_{1}, y_{2}\right)$ subject to the constraint $(-1) \cdot y_{1}+1 \cdot y_{2} \leqq \max _{y_{2} \in[0, b]}\left[p_{2}^{1}+p_{2}^{2}\right] y_{2}=0$. It can also be shown that this Lindahl equilibrium is unique.

Figure 1 illustrates this discussion for the case where $a=b=3$. The dashed lines are indifference curves as labelled. The heavy line is the locus of Pareto efficient points, the segment $\mathrm{AB}$ is the $\alpha$-core and the segment $\mathrm{CD}$ is the set Pareto efficient points which are preferred by both agents to the outcome $(0,0)$. The point $E$ is the only Lindahl equilibrium outcome.

To show that the set of Lindahl equilibria and the $\alpha$-core may be disjoint, consider the case where $a=3$ and $b=2$. According to our previous discussion, the only Lindahl equilibrium outcome is $\left(y_{1}, y_{2}\right)=(1,1)$. For any outcome in the $\alpha$-core, $u^{1}\left(y_{1}, y_{2}\right) \geqq u^{1}(a, b)=u^{1}(3,2)=1$. But $u^{1}(1,1)$ $=\frac{1}{2}$. Thus the (unique) Lindahl equilibrium is not in the $\alpha$-core and we have the promised example. Figure 2 illustrates this case. The Lindahl equilibrium is the point $\mathrm{E}$ and the $\alpha$-core is the segment $\mathrm{AB}$.

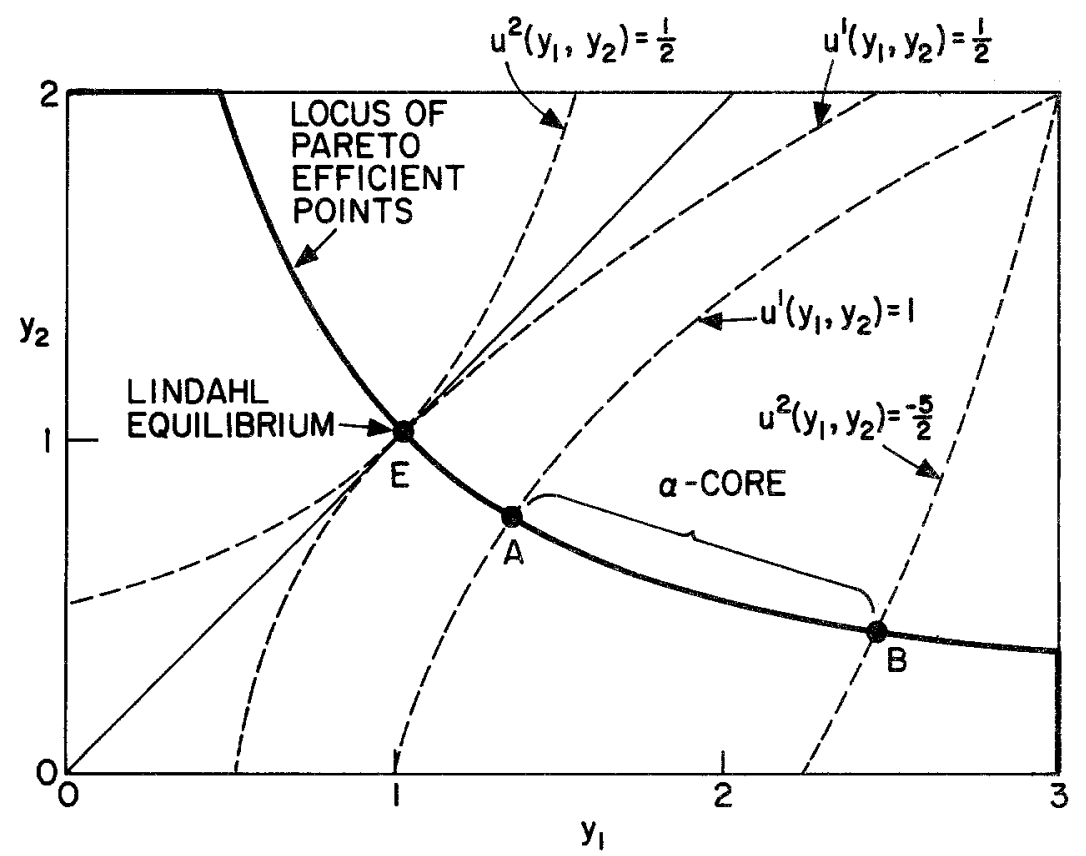

Figure 2. 


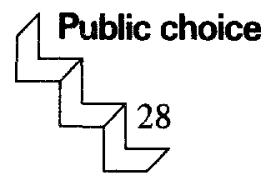

\section{The core when damage is limited by law or technology}

We now consider a fairly strong core property that Lindahl equilibrium does have. While this discussion concerns societies, parallel results for games can be obtained as a straightforward application of Remark 1.

For an $n$ person society, Lindahl prices $\bar{p}=\left(\dot{p}^{1}, \ldots, \bar{p}^{n}\right)$ can be used to compute "values" of the actions of one coalition to another.

Definition 10. Where the actions of the agents are $x_{1}, \ldots, x_{n}$, the benefit value of the actions of coalition $S$ to the complementary coalition, $-S$, is defined as:

$$
\beta_{S}\left(\bar{p}, x_{1}, \ldots, x_{n}\right)=\left({ }_{i \in-S}^{\Sigma} \bar{p}^{i}\right)\left(\sum_{i \in S}^{\Sigma} x_{i}\right) .
$$

It turns out that in Lindahl equilibrium, for every coalition $S$ the benefit value of the actions of $S$ to $\sim S$ equals the benefit value of the actions of $\sim S$ to $S$. Furthermore if another social situation improves on Lindahl equilibrium for some coalition $S$, then in the proposed social situation the benefit value of the actions of $\sim S$ to $S$ exceeds the benefit value of actions of $S$ to $\sim S$. This suggests that Lindahl equilibrium is really a social exchange equilibrium. These results are stated formally in the next remark.

Remark 13. Let $\left(\bar{x}_{1}, \ldots, \bar{x}_{n}, \bar{p}, \ldots, \bar{p}^{n}\right)$ constitute a Lindahl equilibrium for the society $\left(X_{1}, \ldots, X_{n}, R_{1}, \ldots, R_{n}\right)$. If preferences of all agents are locally non-satiated, then:

1. For all $S \subset\{1, \ldots, n\}, \beta_{S}\left(\bar{p}, \bar{x}_{1}, \ldots, \bar{x}_{n}\right)=\beta_{-S}\left(\bar{p}, \bar{x}_{1}, \ldots, \bar{x}_{n}\right)$

2. For all $S \subset\{1, \ldots, n\}$, if $\left(x_{1}, \ldots, x_{n}\right) \in \underset{j=1}{n}{ }_{x}^{n} X_{j}$ and if:

$$
\begin{aligned}
& \sum_{j=1}^{n} x_{j} R_{i}^{S} \sum_{j=1}^{n} \bar{x}_{j} \text { for all } i \epsilon S \text { with strict preference for some } i \epsilon S \text {, then } \\
& \beta_{\sim, S}\left(\bar{p}, x_{1}, \ldots, x_{n}\right)>\beta_{S}\left(\bar{p}, x_{1}, \ldots, x_{n}\right) .
\end{aligned}
$$

Proof:

Let $\left(\bar{p}^{1}, \ldots, \bar{p}^{n}, \bar{x}_{1}, \ldots, \bar{x}_{n}\right)$ be a Lindahl equilibrium and $S \subset\{1, \ldots, n\}$. If preferences are locally non-satiated for all $i \in S$ it follows that $\bar{p}^{i} \sum_{j=1}^{n} \bar{x}_{j}=\left(\sum_{j=1}^{n} \bar{p}^{j}\right) \bar{x}_{i}$ for all $i \epsilon S$. Summing these equations we have $\left(\sum_{i \in S^{5}} \bar{p}^{i}\right) \quad \sum_{j=1}^{n} \bar{x}_{j}=\left(\sum_{j=1}^{n} \bar{p}^{j}\right)\left(\sum_{i \in S}^{\sum} \bar{x}_{i}\right)$. Subtracting $\left({ }_{i \in S}^{\Sigma} \bar{p}^{i}\right)\left(\sum_{i \in S}^{\Sigma} \bar{x}_{i}\right)$ from each side we have $\left(\sum_{i \epsilon S}^{\Sigma} \bar{p}^{i}\right)\left(\sum_{i \epsilon}^{\Sigma} \bar{x}_{i}\right)=\left(\sum_{i \epsilon}^{\Sigma} \bar{p}^{i}\right)\left(\sum_{i \epsilon S}^{\Sigma} \bar{x}^{i}\right)$ which establishes (1). 
If $\left(x_{1}, \ldots, x_{n}\right) \epsilon \underset{j=1}{\stackrel{n}{\times}} X_{j}$ and $\sum_{j=1}^{n} x_{j} R_{i}^{S} \sum_{j=1}^{n} \bar{x}_{j}$ for all $i \in S$ with strict preferences for some $i \in S$, then for all $i \in S, \bar{p}^{i} \sum_{j=1}^{n} x_{j} \geqq\left(\sum_{j=1}^{n} \tilde{p}^{j}\right) x_{i}$ with strict inequality for some $i \in S$. Therefore $\left(\sum_{i \in S}^{\Sigma} \bar{p}^{i}\right)\left(\sum_{j=1}^{n} x_{j}\right)>\left(\sum_{j=1}^{n} \bar{p}^{j}\right)$ $\left(\sum_{i \in S}^{\Sigma} x_{i}\right)$. Subtracting $\left(\sum_{i \in S}^{\Sigma} \bar{p}^{i}\right)\left(\sum_{i \epsilon S}^{\Sigma} x_{i}\right)$ from each side of the inequality we have $\left(\sum_{i \in S}^{\Sigma} \bar{p}^{i}\right)\left(\sum_{i \epsilon \sim}^{\Sigma} x_{i}\right)>\left(\sum_{i \epsilon \sim}^{\Sigma} \bar{p}^{i}\right)\left(\sum_{i \in S}^{\Sigma} x_{i}\right)$. This establishes (2). Q.E.D.

Where $\bar{p}$ is a system of Lindahl prices, we define the set of $\bar{p}$-admissable actions for a coalition $S$ to be those possible actions of coalition $S$ which have non-negative benefit value for $\sim$.

Definition 11. Let $\bar{p}=\left(\bar{p}^{1}, \ldots, \vec{p}^{n}\right)$ be a system of Lindahl prices for the society $\left(X_{1}, \ldots, X_{n}, R_{1}, \ldots, R_{n}\right)$. The set of $\bar{p}$-admissable strategies for $S \subset\{1, \ldots, n\}$ is $\left\{\begin{array}{c}\times \\ i \in S\end{array} x_{i} \in \underset{i \in S}{\times} X_{i} \mid\left(\sum_{i \in \sim S}^{\Sigma} \bar{p}^{i}\right)\left(\sum_{i \in S}^{\Sigma} x_{i}\right) \geqq 0\right\}$.

We now state the promised core property of Lindahl equilibrium.

Remark 14. If preferences of all agents are locally non-satiated, a Lindahl equilibrium $\left(\bar{p}, \bar{x}_{1}, \ldots, \bar{x}_{n}\right)$ has the property that no coalition $S \subset$ $\{1, \ldots, \dot{n}\}$ can improve on $\left(\bar{x}_{1}, \ldots, \bar{x}_{n}\right)$ by means of a strategy which is $\overline{\mathrm{p}}$-admissable for $S$.

\section{Proof:}

If coalition $S$ can improve on $\left(\bar{x}_{1}, \ldots, \bar{x}_{n}\right)$, then there is some $x_{S}^{\prime}=\sum_{i \in S} x_{i}^{\prime}$ $\epsilon \underset{i \in S}{\Sigma} X_{i}$ such that for all $x \sim \sim_{S}^{\prime} \epsilon \underset{i \epsilon \sim S}{\Sigma} X_{i}$, and for all $j \in S,\left(x_{j}^{\prime}, x_{S}^{\prime}+x_{\sim \mathcal{S}}^{\prime}\right) R_{j}$ $\left(\bar{x}_{j}, \sum_{i \in S} \bar{x}_{i}^{\prime}\right)$ with strict preference from some $j \epsilon S$. From Remark 15 it then follows that $\left(\sum_{i \in S}^{\Sigma} \bar{p}^{i}\right)\left(\sum_{i \in S^{2}}^{\Sigma} x_{i}^{\prime}\right)>\left(\sum_{i \in \sim}^{\Sigma} \bar{p}^{i}\right)\left(\sum_{i \in S}^{\Sigma} x_{i}^{\prime}\right)$. Since, by construction, $0-X_{i}$ for all $i$, this inequality must hold when $x_{i}^{\prime}=0$ for all $i \in \sim S$. Therefore $\left(\sum_{i \in \sim S}^{\Sigma} \bar{p}^{i}\right)\left(\sum_{i \in S}^{\Sigma} x_{i}^{\prime}\right)<0$. But this means that the choice of strategies $x_{i}^{\prime}$ for members of $S$ is not $\overline{\mathrm{p}}$-admissable for $S$. Q.E.D.

We conclude by observing some examples of restrictions on strategies which if imposed either by technology or by law will restrain all coalitions to $\bar{p}$-admissable strategies where $\bar{p}$ is any system of Lindahl prices. 


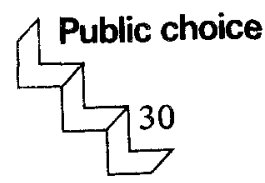

\section{Restriction 1}

For all agents, $X_{i}$ is the non-negative orthant of Euclidean $n$-space and preferences are monotone non-decreasing in each component. Then any Lindahl price system $\bar{p}$ must be non-negative and hence all feasible strategies must be $\overline{\mathrm{p}}$-admissable. This is the case of external economies discussed by Shapley and Shubik (1969).

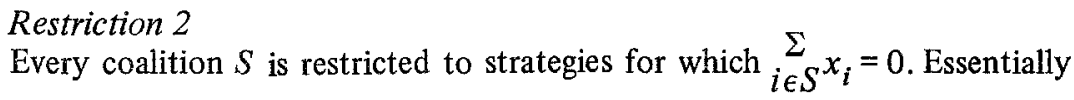
what this restriction requires is that although coalitions may rearrange levels of actions among their members, they are not allowed to impose effects on non-members other than the effects imposed by the "zero" actions. This restriction is suggested by Starrett (1973).

Restriction 3
Each coalition $S$ is restricted to actions $x_{i}$ for $i \in S$ such that $x+\sum_{i \in S} x_{i} R_{j} x$ for all $j \in \sim S$ and all $x \in \underset{j \in \sim S}{\Sigma} x_{j}$. If $\bar{p}$ is a system of Lindahl prices then all actions which comply with these restrictions will be $\overline{\mathrm{p}}$-admissable for $S$. This result is due to Bergstrom (1975). An example of a system of restrictions of this kind would be a society of agents living on a river and discharging effluents as well as engaging in trade in ordinary private goods. If the only "externalities" in the system are due to the downstream flow of effluents, then any coalition which excludes no one living downstream from its members can be left free to perform all exchanges and discharges which are technically feasible. In this case, a restriction of type 3 is very simply articulated and leaves a rather rich structure of coalitions free to perform without restriction.

\section{Notes}

1. The condition $0 \epsilon Y$, can be imposed without loss of generality just by translation.

2.

The set $\underset{i=1}{\mathrm{x}} Y_{i}$ is the cartesian product of the sets $Y_{1}, \ldots, Y_{n}$.

3.

The $X_{i}$ is now the same as the $Y_{i}$ in Rader (1976) since the origin is specified. We can now use $Y_{i}$ for the traditional game notation.

4.

The set $\Sigma X_{i}$ is the set $\left\{\mathrm{x} / \mathrm{x}=\sum_{i=1}^{n} x_{i}\right.$ where $x_{i} \in X_{i}$ for $\left.i=1, \ldots, n\right\}$. 


\section{References}

Arrow, K. J., "The Organization of Market Activity: Issues Pertinent to the Choice of Market Versus Nonmarket Allocation," in Joint Economic Committee, Analysis and Evaluation of Public Expenditures: The PPB System, Washington, D. C.: U. S. Government Printing Office, 1969, pp 47-64.

Bergstrom, T. C., "Collective Choice and the Lindahl Allocation Method," in Lin, S. Y. (ed), Economics of Externalities, New York: Academic Press 1976. "The Core When Strategies are Restricted by Law," Review of Economic Studies, 42, April 1975, pp. 248-257.

Debreu, G., The Theory of Value, New York: Wiley, 1959.

Nikaido, H., Convex Structures and Economic Theory, New York: Wiley, 1968.

Rader, J. T., "An Economic Approach to Social Choice," Public Choice, 15, 1973, pp. 49-75.

Press, 1972 .

Theory of General Economic Equilibrium, New York: Academic

Scarf, H., "On the Existence of a Cooperative Solution for a General Class of $N$-person Games," Journal of Economic Theory, 3, 1971, pp. 169-181.

Shapley, L. and Shubik, M., "On the Core of an Economic System with Externalities," American Economic Review, 59, 1969, pp. 678-684.

Starrett, D., "A Note on Externalities and the Core," Econometrica , 41, 1973, pp. 179-183. 